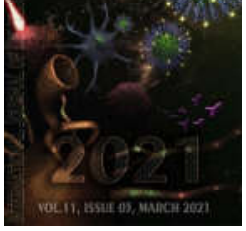

\title{
PRINCIPAIS CAUSAS DA NÃO ADESÃO AO ALEITAMENTO MATERNO EXCLUSIVO NO BRASIL: REVISÃO INTEGRATIVA DE LITERATURA
}

\author{
Ana Carolina da Silva Soares Martins ${ }^{1}$, Ilma Terezinha Palmeira Teixeiraㄹ, Mariane Costa Santos de \\ Tavares $^{3}$, Indira da Natividade Leão Costa ${ }^{4}$, Gilmária Ferreira de Almeida ${ }^{5}$, Maria Augusta Cunha \\ Menezes $^{6}$, Rosy Aline Lopes de Oliveira ${ }^{6}$, Poliana Terra Pires Ribeiro Coelho Caires ${ }^{6}$, Kalile dos Anjos \\ Souza $^{6}$ and Laís Guedes Rodrigues ${ }^{6}$
}

1Discente do Curso de Graduação em Medicina Faculdades Santo Agostinho, Vitória da Conquista - BA, Brasil; ${ }^{2}$ Médica Pediatra. Docente do Curso de Graduação em Medicina, Faculdades Santo Agostinho, Vitória da Conquista - BA, Brasil; ${ }^{3}$ Fisioterapeuta. Especialista em Fisioterapia Respiratória e Terapia Intensiva pelas Faculdades Integradas Pitágoras, Pós -

Graduada em Deficiências Múltiplas e Sensoriais. Discente do Curso de Graduação em Medicina Faculdades Santo Agostinho, Vitória da Conquista - BA, Brasil; ${ }^{4}$ Biomédica. Discente do Curso de Graduação em Medicina Faculdades Santo Agostinho, Vitória da Conquista - BA, Brasil; ${ }^{5}$ Médica. Pós-Graduada em Atenção Básica pela Universidade Federal do Maranhão - UFMA, São Luís - MA, Brasil; ' ${ }^{D}$ iscente do Curso de Graduação em Medicina Faculdades Santo Agostinho, Vitória da Conquista - BA, Brasil

\section{ARTICLE INFO}

\section{Article History:}

Received $02^{\text {nd }}$ January, 2021

Received in revised form

$27^{\text {th }}$ January, 2021

Accepted $10^{\text {th }}$ February, 2021

Published online $30^{\text {th }}$ March, 2021

\section{Key Words:}

Breastfeeding. Child nutrition.

Child health.

\begin{abstract}
Exclusive breastfeeding is of fundamental importance for the neuropsychomotor development of the child, as well as for the maintenance of their health. The aim of the study proposed here was to evaluate the reasons for the abandonment of exclusive breastfeeding in Brazil, described in the scientific literature. This is an integrative literature review, carried out in the databases linked to the Virtual Health Library (VHL), Scientific Electronic Libray Online (SciELO), Latin American and Caribbean Literature/Bibliographic Index EspañolenCiencias de la Salud (Lilacs/Ibecs), and Medical Literature Analysis and Retrievel System Online (Medline). The search was conducted with the descriptors in health sciences (DECS): "Breastfeeding" AND "Child nutrition", "Breastfeeding" AND "Child health", "Breastfeeding" OR "Breastfeeding". "Child nutrition" AND "Infant". Given the relevance of breastfeeding for child development, it is understood that from the understanding of possible reasons that lead to low adhering or abandonment of breastfeeding, it is possible to devise strategies for coping with such obstacles. The existence of myths about breastfeeding, the woman's need to return to work, as well as deficient knowledge about the importance of exclusive breastfeeding were the main reasons mentioned in the literature for non-smoking. The protective factors of exclusive breastfeeding were mentioned: promotion of breastfeeding during prenatal care, puerperal home visit, as well as family support and greater knowledge of women about the benefits of the practice.
\end{abstract}

*Corresponding author:

Ana Carolina da Silva Soares Martins

Copyright (C) 2021, Ana Carolina da Silva Soares Martins et al. This is an open access article distributed under the Creative Commons Attribution License, which permits unrestricted use, distribution, and reproduction in any medium, provided the original work is properly cited.

Citation: Ana Carolina da Silva Soares Martins, Ilma Terezinha Palmeira Teixeira, Mariane Costa Santos de Tavares, Indira da Natividade Leão Costa, Gilmária Ferreira de Almeida, Maria Augusta Cunha Menezes, Rosy Aline Lopes de Oliveira, Poliana Terra Pires Ribeiro Coelho Caires, Kalile dos Anjos Souza6 and Laís Guedes Rodrigues, 2021. "Principais causas da não adesão ao aleitamento materno exclusivo no Brasil: revisão integrativa de Literatura", International Journal of Development Research, 11, (03), 45619-45623.

\section{INTRODUÇÃO}

O Aleitamento Materno (AM) é descrito pela literatura como uma estratégia fundamental para redução da morbimortalidade infantil (BRASIL, 2013). O Aleitamento Materno Exclusivo (AME), por sua vez, pode ser compreendido como a alimentação do bebê exclusivamente com leite materno até os seis meses de vida, sendo posteriormente mantido o AM até os dois anos de idade, sendo complementado nesta segunda etapa por outros alimentos (ARAÚJO et al., 2013).
O aleitamento materno ofertado é considerado a primeira prática relacionada a nutrição da criança e que é estimulada para que seja praticada de forma exclusiva. Esta prática é de fundamental importância para a prevenção de doenças e também para a promoção da saúde da mãe e do recém-nascido. Além de conferir nutrição e proteção, o aleitamento materno é ainda capaz de influenciar todo o estado fisiológico, emocional e cognitivo da criança e da mãe, sendo considerado então, um profundo processo de interação (JENERAL et al., 2015). A prática do aleitamento esteve presente durante todo o desenvolvimento da humanidade, supondo que por esta concepção 
epigenética, o leite materno permitiu que o potencial genético próprio dos seres humanos fosse atingido com êxito. Isto se deve ao fato de que a composição nutricional do leite materno possui quantidades suficientes de nutrientes como proteínas, lipídios, carboidratos e água. Além de conferir a nutrição, a prática confere ainda proteção imunológica e auxilia no desenvolvimento de habilidades sem que seja necessária a adição de outras fontes de nutrição nos primeiros seis meses de vida (AMARAL et al., 2015). Intimamente relacionado com a diminuição da morbidade e da mortalidade infantil, o aleitamento materno é importante por reduzir as chances da criança de contrair infecções respiratórias, intestinais, infectocontagiosas e alergias alimentares. Nunes (2015) afirma ainda que a prática do aleitamento materno é capaz de prevenir cerca de $13 \%$ dos óbitos evitáveis em crianças com idade inferior a cinco anos. $\mathrm{O}$ aleitamento materno salvaria em torno de 1,47 milhões de vidas por ano em todo o mundo, caso a recomendação de ofertar de forma exclusiva o leite materno a criança até os seis meses fosse seguida. Outros benefícios desta prática, incluem a diminuição das mortes súbitas do lactente, alergias, dermatite atópica e asma brônquica. Influenciam ainda no desenvolvimento do crânio e das funções orais, mastigatórias e de fala, auxiliando ainda a escolarização (NUNES, 2015). Ademais, o próprio ato de sucção realizado pela criança para retirar o leite materno é importante para o desenvolvimento anatômico e fisiológico da cavidade oral, de modo a alinhar o palato duro, os dentes e oferecer uma oclusão dentária adequada. Por isto, que a interrupção precoce do aleitamento materno é prejudicial para a cavidade oral, impactando diretamente na respiração, deglutição, articulação de sons de forma motora, além de provocar oclusões dentárias. A utilização de chupetas e mamadeiras potencializa estas alterações motoras e orais (ROCHA; COSTA, 2015). Segundo a Organização Mundial da Saúde (OMS, 2007) o aleitamento materno é descrito quando a criança recebe leite materno direto da mama, ou por ordenha, e pode ser classificado em quatro categorias:

- Aleitamento materno exclusivo: em que a alimentação do lactente se dá exclusivamente pelo leite materno, direto da mama, por ordenha, ou ainda leite materno de outra fonte, como bancos de leite humano. Nesta modalidade não há oferta de outros líquidos ou sólidos. Com exceção de medicamentos, vitaminas, suplementos minerais ou sais de reidratação que se façam necessários.

- Aleitamento materno predominante: trata-se do contexto alimentar em que a criança, além do leite materno recebe água, chás, sucos de frutas, dentre outras bebidas, mas ainda há predomínio do aleitamento materno como fonte nutricional.

- Aleitamento materno complementado: quando o lactente recebe o leite materno, por ordenha ou mama, e recebe de forma complementar alimento sólido ou semissólido, com o intuito de complementação do aleitamento e não substituição.

- Aleitamento materno parcial ou misto: situação em que a criança recebe além de leite materno outros tipos de leite.

Vieira et al. (2015) afirmam que o crescimento nos primeiros meses de vida tem sido objeto de diversos estudos por acreditar-se que alterações do processo de crescimento possam provocar atrasos no desenvolvimento neuropsicomotor, com consequente aumento do risco de morbimortalidade infantil. Em países como o Brasil, verificou-se a redução da velocidade de crescimento, principalmente nos primeiros seis meses de vida. Existem indicativos que tal redução possa ser causada pela substituição do leite materno por outros alimentos que não possuam o mesmo valor nutricional. Além disso, com a interrupção da amamentação a criança pode ser exposta a alimentos contaminados elevando sua vulnerabilidade a infecções. $\mathrm{O}$ Ministério da Saúde recomenda que o aleitamento materno seja oferecido de forma exclusiva até os seis meses de idade a livre demanda e perdure até os dois anos de idade. Isto ocorre, pois, a introdução alimentar de forma precoce pode impactar na saúde da criança, sendo deletéria para seu desenvolvimento. Entre os principais problemas de uma introdução alimentar precoce, há os maiores riscos de episódios diarreicos, menor absorção de nutrientes importantes para o funcionamento metabólico como o zinco e o ferro, menor duração do aleitamento materno e desnutrição acentuada (BRASIL,
2015). Em estudo desenvolvido por Rollins et al. (2016) os pesquisadores avaliaram o aleitamento materno em 153 países, incluindo o Brasil. Verificou-se que as crianças brasileiras apresentaram um importante acréscimo no tempo de aleitamento materno. No ano de 1975 o tempo de amamentação médio era de 2,5 meses, em 2006 verificou-se um tempo médio de 14 meses de aleitamento, o que representa um acréscimo de $15 \%$ no tempo de aleitamento. Silva et al. (2019) apontam que a alimentação da criança em seu primeiro ano de vida irá influenciar seu metabolismo e crescimento ao longo da vida. Segundo os autores a interrupção do aleitamento, e introdução precoce de alimentação complementar, pode propiciar carências nutricionais, comprometimento do desenvolvimento, bem como, a ocorrência de doenças e agravos. No estudo, crianças amamentadas tiveram maior ingestão de vitamina A, e melhor aporte nutricional ao longo do primeiro ano de vida.

A primeira secreção láctea produzida após o parto é denominada "leite colostro", tal leite permanece até o décimo dia pós -parto, sendo espesso, de alta densidade, com elevado teor de carotenoides. O colostro é rico em anticorpos, o que confere proteção contra infecções ao recém-nascido, além de ser um composto de fácil digestão e contributivo para estabelecimento da flora intestinal do neonato (BOCCOLINI et al., 2013). Entre o oitavo e décimo quinto dia pós-parto, inicia-se a liberação do leite de transição, e posteriormente, após cerca de duas semanas pós-parto, tem-se o leite maduro. Há diferenças significativas entre as fases do leite materno e sua composição. Enquanto o colostro possui maior concentração proteica, no leite maduro, verifica-se maior aporte gorduroso . A concentração de vitamina $\mathrm{E}$ no leite humano, também é observada em maior proporção no colostro que no leite maduro (LIMA; DIMENSTEIN; RIBEIRO, 2014). Durante uma mesma mamada há ainda alterações na concentração de gordura no leite. No início da mamada, verifica-se um leite com menor teor gorduroso, enquanto o leite posterior possui maior aporte energético, o que garante a saciedade da criança, e revela a importância de se orientar o esvaziamento completo da mama (BRASIL, 2009). A digestibilidade do leite humano também é significativamente maior, que o leite de vaca, pelas proteínas encontradas e capacidade de degradação enzimática do sistema digestório do bebê (BRASIL, 2009). Santos et al (2015) evidenciaram que o leite materno tem sido uma importante estratégia das políticas públicas para melhorar a situação de saúde da população infantil, de modo a reduzir a mortalidade e a morbidade, impactando de forma direta na melhoria dos indicadores de saúde. Desta forma, os benefícios do aleitamento implicam também na redução das taxas de diarreia, sendo esta, considerada um grave problema de saúde pública, responsável pela maior parte das internações hospitalares infantis, precedida somente das infecções respiratórias, que são a principal causa de óbito (SANTOS et al., 2015).

O benefício financeiro também deve ser considerado, pois o leite materno é a forma mais econômica de nutrição e pronto para ser servido à livre demanda e em temperatura ambiente. Apesar de não ser considerado estéril, as crianças nascidas a termo e sem complicações são capazes de tolerar uma quantidade variada de microrganismos sem patogenicidade importante, adicionando então, vantagens em relação a menor quantidade de consultas (VILLAÇA; FERREIRA; WEBER, 2015). Por este motivo, é importante que o aleitamento materno seja oferecido de forma exclusiva para a criança e de forma humanizada. Para isto, a Organização Mundial da Saúde e o Fundo das Nações Unidas para a Infância orienta que a amamentação seja realizada de forma humanizada por meio dos dez passos para o sucesso do aleitamento materno. Esta política atua como forma norteadora para capacitar as equipes de saúde, auxiliar a amamentação na primeira hora de vida, ofertar conhecimento às gestantes acerca da importância do aleitamento materno oferecido de forma exclusiva, bem como os benefícios para a sua própria saúde e para o recém-nascido, além de estimular a lactação, oferecer suporte, apoio e incentivo a prática e esclarecer sobre não ser necessário adicionar chás e água a dieta da criança (BRASIL, 2019). Ouchi et al (2017) discutem acerca dos benefícios sociais que podem estar associados com a prática do aleitamento materno exclusivo. Estes 
benefícios podem ser estimados por meio dos indicadores de saúde, redução da mortalidade infantil e morbidades. Além disto, o aleitamento faz com que as crianças adoeçam menos, reduzindo assim, as hospitalizações, a utilização de fármacos, consultas médicas e a ausência dos pais em atividades laborais diárias (OUCHI et al., 2017). Além dos diversos benefícios conferidos a mãe e a criança relacionados a nutrição, sistema imune e prevenção de cânceres, a amamentação é capaz ainda de fortalecer o vínculo entre a mãe e o recém-nascido, impactando diretamente no desenvolvimento biopsicossocial da criança. Por isto, o processo deve ser considerado uma experiência única e particular em que o foco não deve ser destinado apenas a criança, mas envolver toda a família. Recomendase ainda que todo recém-nascido seja colocado no peito da mãe para que a primeira mamada aconteça após o parto para estreitar ainda mais os laços e aumentar o vínculo entre mãe e filho (CARNEIRO et al., 2014). De acordo com Caminha et al. (2014) o Baixo Peso ao Nascer (BPN) é uma condição caracterizada pela presença de um nascido vivo com peso inferior à $2.500 \mathrm{~g}$. $\mathrm{O} \mathrm{BPN}$ está relacionado à mais de $50 \%$ dos óbitos nos primeiros anos de vida. Uma das estratégias para garantir à neonatos com BPN um bom desenvolvimento e a recuperação adequada do peso corporal é assegurar ao bebê o AME. Conforme afirmado por Boccolini et al. (2011) estimular o aleitamento materno no âmbito da APS é garantir que a criança tenha acesso ao único nutriente capaz de garantir seu crescimento normal e adequado, reduzindo ainda risco de morte por diarreias, reações alérgicas e internações hospitalares. Neste contexto, o presente estudo buscou analisar na literatura possíveis motivos que levam à baixa adesão ou ao abandono do aleitamento materno exclusivo no Brasil. Para alcançar os objetivos propostos foi estruturada a seguinte questão de pesquisa: quais os motivos podem levar ao abandono do aleitamento materno exclusivo e suas consequências no contexto brasileiro?

Diante da relevância do AME para o bom desenvolvimento neuropsicomotor da criança, entende-se que a partir da compreensão de possíveis motivos que levem à baixa adesão ou ao abandono do AME torna-se possível traçar estratégias de enfrentamento de tais obstáculos. Desta forma, o estudo se justifica, exatamente, por propor a identificação e discussão de possíveis determinantes da má adesão ao AME.

\section{MATERIAIS E MÉTODOS}

$\mathrm{O}$ estudo caracteriza-se como uma pesquisa de revisão integrativa da literatura.A estratégia adotada para a obtenção dos dados deste trabalho foi a pesquisa bibliográfica. Os bancos de dados pesquisados foram a Biblioteca Virtual em Saúde (BVS), Scientific Electronic Libray Online (SciELO), Literatura Latino-Americana e do Caribe/Índice Bibliográfico EspañolenCiencias de laSalud (Lilacs/Ibecs), e Medical LiteratureAnalysis and Retrievel System Online (Medline) .Os descritores de ciências da saúde (DECS) utilizados para a busca foram: "Aleitamento materno" AND "Nutrição da criança", "Amamentação" AND "Saúde da criança", "Aleitamento materno" OR "Amamentação". "Nutrição da criança" AND "Lactente". Após a pesquisa foi realizada uma análise do material encontrado, selecionando as publicações que se adequavam aos objetivos do trabalho. Foram incluídos estudos originais, publicados preferencialmente em língua portuguesa, visto que se busca a análise do contexto nacional. Caso publicados por autores brasileiros nos idiomas inglês e espanhol, artigos em tais idiomas também foram considerados.Por ser um estudo de revisão literária, e com uso restrito de dados secundários, a presente pesquisa foi dispensada da submissão ao Comitê de Ética e pesquisa, conforme resolução $n^{\circ}$ 466, de 12 de dezembro de 2012 (BRASIL, 2012).

\section{RESULTADOS E DISCUSSÃO}

O aleitamento materno exclusivo é de importância para o recémnascido, mas também é de grande relevância para a mãe. É neste momento em que os laços afetivos começam a se solidificar, devido ao contato pele a pele, ao olhar e ao momento que é dedicado ao cuidado entre mãe e filho. Além disso, a amamentação após o parto protege a mãe de adquirir anemias, sangramentos e auxilia no retorno da musculatura uterina para posição inicial, através da estimulação da produção de hormônios, como a prolactina que será fundamental na produção de leite, e a ocitocina que promove a liberação do leite e faz o útero se contrair. A prática confere ainda, proteção a mãe, diminuindo as chances de desenvolver câncer ovariano e uterino (SILVA et al., 2016). A excelência e os benefícios do aleitamento materno são incontestáveis, apesar de toda a influência da cultura, hábitos, mitos e crenças particulares a cada indivíduo. O aleitamento é considerado a mais importante e sábia estratégia orgânica de intervenção para diminuição da morbidade infantil. Sendo ainda fundamental para estabelecer vínculo, afeto, economia, proteção, nutrição e aproximação entre mãe e filho, o aleitamento é capaz de prevenir problemas como gastroenterites e estimular o crescimento e o desenvolvimento de forma saudável (SILVA; GOETZ; SANTOS, 2017). De acordo com Melo et al. (2017) o leite materno além de ser um alimento completo garante ao bebê uma segunda linha de defesa imunológica enquanto seu próprio sistema de defesa ainda se encontra imaturo. Os autores ponderam que a questão do aleitamento materno deve ser inserida nas discussões entre profissionais de saúde e gestantes desde o primeiro contato no pré-natal, estimulando assim não apenas a aceitação do ato do aleitamento, mas o desejo de amamentar. Dentre os fatores promotores do AME foram citados no estudo: alojamento conjunto além do apoio para amamentar por profissionais e familiares. As principais dificuldades encontradas no aleitamento foram a dor, fissuras mamilares e mastite. O abandono do AME foi feito com inserção de outros leites industrializados, água, chás.

Estudos demonstram que quando incentivado ainda durante o prénatal as taxas de aleitamento materno exclusivo aumentam consideravelmente. A equipe da Atenção Básica deve estar prontamente capacitada para orientar precocemente as gestantes acerca dos benefícios da prática do aleitamento materno. O profissional da saúde assume, então, um papel regulador da prática do aleitamento materno (VILLAÇA; FERREIRA; WEBER, 2015). Vieira et. al. (2015) realizaram um estudo com 371 lactentes do município de Viçosa/MG, onde verificou-se que 3,5\% da amostra apresentava baixo peso, e $3,7 \%$ apresentava baixo comprimento para a idade. $O$ peso elevado foi encontrado em $2,4 \%$ da amostra. Apenas $20,8 \%$ da amostra teve AME. De acordo com os autores a AME ou predominante nos primeiros seis meses de vida está associada a um maior ganho de peso. A participação das mães em todo o acompanhamento pré-natal também foi apontada como fator que corrobora com a adesão ao AME. Em estudo realizado por Ferreira et al. (2018) os pesquisadores apontam forte correlação entre multiparidade e manutenção do AME. Por outro lado, mães adolescentes, e que não participaram adequadamente do acompanhamento pré-natal tiveram menor adesão ao AME. Entre as mulheres que amamentou exclusivamente verificou-se ainda um relato comum de baixa orientação sobre as práticas e importância do aleitamento materno durante as consultas de pré-natal. Segundo as mesmas, o apoio da família e materiais divulgados pela mídia foram decisivos na decisão do AME.

Outra estratégia apontada como motivador do AME é a primeira visita domiciliar puerperal. Do total de 62 crianças acompanhadas por estudo realizado no Estado de Pernambuco/Brasil, verificou-se uma adesão ao AME de 41,7\%. Variáveis como renda familiar, multiparidade, hábito de chupeta e peso ao nascer não apresentaram significância estatística sobre a manutenção do AME. Contudo, entre as mulheres que receberam a visita puerperal houve maior empenho e adesão ao AME. Conforme os pesquisadores a visita domiciliar pela equipe de Atenção Básica no puerpério imediato aumenta o vínculo com a puérpera, permite identificar dificuldades no aleitamento de forma precoce, bem como, se estabelece momento educativo e de promoção de hábitos saudáveis e cuidados adequados ao recémnascido e à puérpera (CARVALHO et al., 2018). É importante que o aleitamento materno seja baseado no envolvimento de toda a família, pois é um momento de construção de afetos e pode auxiliar no 
prolongamento da prática e também acaba naturalizando o planejamento familiar. Caso ainda não tenha ocorrido períodos menstruais, o aleitamento materno exclusivo diminui as chances de nova gravidez, pois o organismo materno continua a produzir hormônios que inibem a ovulação (CONCEIÇÃO et al., 2015). Lima et al. (2019) investigaram os motivos para interrupção do AME em prematuros no primeiro mês após a alta hospitalar. No estudo a prevalência de AME na alta foi de $85,2 \%$, decaindo para $75 \%$ após 15 dias e para $46,3 \%$ aos 30 dias. O principal motivo relatado foi a "insuficiência do leite" e a crença de "leite fraco". Diante dos resultados obtidos os pesquisadores ressaltam a importância do acompanhamento pós-alta para reduzir o desmame precoce, com orientação adequada às lactantes e desmistificação do AME. A existência de crenças e mitos sobre o aleitamento materno também foi referida por Carreiro et al. (2018) como um dos principais motivos para o abandono do AME. A percepção das mães sobre a quantidade de leite produzido, dificuldades no aleitamento, e ocorrência de fissuras mamilares também foram referidos. Entre os fatores protetores do AME citados destacam-se o contato precoce pele a pele entre puérpera e bebê, maior escolaridade, situação conjugal estável, extração do leite de forma manual com facilidade, além do maior apoio familiar.

\section{CONCLUSÃO}

A partir dos dados encontrados na literatura pode-se inferir que o abandono do AME possui etiologia multifatorial. Desde dificuldades maternas com o aleitamento como a ocorrência de fissuras em mamilos, até crenças e mitos sobre produção de leite insuficiente, ou "leite fraco". O papel da Atenção Primária à Saúde foi ressaltado em diversos estudos, seja na abordagem e promoção do AME durante o acompanhamento pré-natal, como também na realização de visita domiciliar puerperal. A lactante com apoio familiar, bem instruída, e que consegue associar sua rotina ao aleitamento comumente consegue manter a prática exclusiva até os seis meses de idade do bebê, e o aleitamento complementado ou predominante até dois anos de idade. Por outro lado, mulheres que precisam retornar ao trabalho, que não recebem apoio familiar, ou que não foram orientadas sobre a importância do AME tendem a abandonar tal prática antes dos quatro meses de aleitamento.

\section{REFERÊNCIAS}

AMARAL, L. J. X. et al. Fatores que influenciam na interrupção do aleitamento materno exclusivo em nutrizes. Revista Gaúcha de Enfermagem, [S. l.], p. 127-34, 2015.

ARAÚJO, N.L. et al. Alimentação dos lactentes e Fatores relacionados ao aleitamento materno.

BOCCOLINI, C.S. et al. Breastfeeding during the first hour of life and neonatal mortality. J Pediatr., v.89, p.131-6, 2013.

BOCCOLINI, C.S. et al. O papel do aleitamento materno na redução das hospitalizações por pneumonia em crianças brasileiras menores de 1 ano. J Pediatr, v.87, n.5, p.399-404, 2011.

BRASIL, Ministério da Saúde. Saúde da criança: aleitamento materno e alimentação complementar. Cadernos de Atenção Básica, Brasilia, n. 2, p. 17-23, 2015.

BRASIL. Ministério da Saúde (BR). Secretaria de Atenção à Saúde. Departamento de Atenção Básica. Manual instrutivo das ações de alimentação e nutrição na Rede Cegonha. Brasília (DF): Ministério da Saúde; 2013.

BRASIL. Ministério da Saúde. Secretaria de Atenção à Saúde. Departamento de Atenção Básica. Saúde da criança: nutrição infantil: aleitamento materno e alimentação complementar / Ministério da Saúde, Secretaria de Atenção à Saúde, Departamento de Atenção Básica. - Brasília : Editora do Ministério da Saúde, 2009. 112 p. : il. - (Série A. Normas e Manuais Técnicos) (Cadernos de Atenção Básica, n. 23)

BRASIL. Ministérios da Saúde. Conselho Nacional de Saúde. Resolução $\mathrm{n}^{\circ}$ 466, de 12 de dezembro de 2012. Aprovar diretrizes e normas regulamentadoras de pesquisas envolvendo seres humanos. Diário Oficial [da] República Federativa do Brasil, Brasília, DF, 13 jun. 2013. Seção 1, p. 59-62.

CAMINHA, M.F.C. et al. Aleitamento materno em crianças de 0 a 59 meses no Estado de Pernambuco, Brasil, segundo o peso ao nascer. Ciênc. saúde coletiva, v.19, n.7, p. 2021-2032, 2014.

CARNEIRO, L. M. de M. C. et al. Prática do aleitamento materno por puérperas: fatores de risco para o desmame precoce. Disciplinarum Scientia. Série: Ciências da Saúde, Santa Maria, v. 15, n. 2, p. 239-248, 2014.

CARREIRO, J.A. et al. Dificuldades relacionadas ao aleitamento materno: análise de um serviço especializado em amamentação. Acta Paul Enferm, v.31, n.4, p.430-438, 2018.

CARVALHO, M.J.L.N. et al. Primeira visita domiciliar puerperal: uma estratégia protetora do aleitamento materno exclusivo. Rev. paul. pediatr., São Paulo, v. 36, n. 1, p. 66-73, Mar. 2018 .

CONCEICAO, S. P. da; FERNANDES, R. A. Q.. Influência da gravidez não planejada no tempo de aleitamento materno. Esc. Anna Nery, Rio de Janeiro, v. 19, n. 4, p. 600-605, dez. De 2015.

DADALTO, E. et al. Conhecimento sobre os benefícios do aleitamento materno e desvantagens da chupeta relacionados a pratica das mães ao lidar com recém nascidos pré- termo. Revista Paulista de Pediatria, [S. l.], p. 399-406, 23 fev. 2017.

FERREIRA, H. L. O. C. et al . Fatores Associados à Adesão ao Aleitamento Materno Exclusivo. Ciênc. saúde coletiva, Rio de Janeiro, v. 23, n. 3, p. 683-690, Mar. 2018.

JENERAL, R. B. R. et al. Aleitamento materno: uma reflexão sobre o papel do pai. Rev. Fac. Ciênc. Méd. Sorocab, [S. l.], v. 17, n. 3, p. $140-147,2015$.

LIMA, A. P. E. et al . Aleitamento materno exclusivo de prematuros e motivos para sua interrupção no primeiro mês pós-alta hospitalar. Rev. Gaúcha Enferm., Porto Alegre , v. 40, e20180406, 2019.

LIMA, M. S.R.; DIMENSTEIN, R.; RIBEIRO, K. D.S. Concentração de vitamina $\mathrm{E}$ no leite humano e fatores associados: uma revisão de literatura,. J. Pediatr. v. 90, n. 5, p. 440-448, Oct. 2014

MELO, R.S. et al. Práticas de Aleitamento Materno Exclusivo entre profissionais de saúde de um hospital Amigo da Criança. Cogitare Enferm. v.22, n.4, p.1-10, 2017.

NUNES, L. Importância do aleitamento materno na atualidade. Boletim Científico de pediatria, Rio de Janeiro, p. 55-58, 18 fev. 2015.

OUCHI, J. et al. Importância da Enfermeira na Orientação da Gestante e Puérpera sobre Aleitamento Materno. Ensaios Cienc., Cienc. Biol. Agrar. Saúde, [S. l.], v. 21, n. 3, p. 134-141, 20 dez. 2017.

PEREIRA, R. et al. Fatores associados ao aleitamento materno exclusivo: o papel do cuidado na atenção básica. Caderno de Saúde Pública, Rio de Janeiro, p. 2343-2354, 15 dez. 2010.

ROCHA, M. G.; COSTA, E. S.. Interrupção precoce do aleitamento materno exclusivo: experiências com mãe de crianças em consulta de puericultura. Rev Bras Promoç Saúde, Fortaleza, v. 28, n. 4 , p. 547-552, 2015.

ROLLINS, N. C. et al. Why invest, and what it will take to improve breastfeeding practices? Lancet, v. 387 , n. 10017, p. 491-504, Jan 302016

SANTOS , F. S. et al. Aleitamento materno e proteção contra diarreia: revisão integrativa da literatura. Einstein (São Paulo), São Paulo, v. 13, n. 3, p. 435-440, 2015.

SILVA , K. M. dos S.; GOETZ , E. R.; SANTOS , M. V. J.. Aleitamento Materno: Conhecimento das Gestantes Sobre a Importância da Amamentação na Estratégia de Saúde da Família. Revista Brasileira de Ciências da Saúde, Fortaleza, v. 21, n. 2, p. 111-118, 2017.

SILVA, C. M. e et al . Fatores associados ao contato pele a pele entre mãe/filho e amamentação na sala de parto. Rev. Nutr., Campinas, v. 29, n. 4, p. 457-471, Aug. 2016.

SILVA, M. A. et al . Relação entre os tipos de aleitamento materno e o consumo de vitamina A e ferro em crianças de 6 a 12 meses. Ciênc. saúde coletiva, Rio de Janeiro, v. 24, n. 11, p. 4009-4018, 2019 
VIEIRA, S. A. et al . Fatores associados às velocidades de ganho de peso e de comprimento nos primeiros seis meses de vida. Cad. saúde colet., Rio de Janeiro, v. 23, n. 3, p. 309-315, Sept. 2015.

VILLAÇA, L. M. de S.; FERREIRA, A. G. da S.; WEBER, L. C.. A importância do aleitamento materno para o binômio mãe-filho disponibilizado pelo banco de leite humano. Rev Saúde AJES, Fortaleza, v. 1, n. 2, p. 1-19, 2015.
WORLD HEALTH ORGANIZATION (WHO). Indicators for assessing infant and young child feeding practices : conclusions of a consensus meeting held 6-8 November 2007 in Washington D.C., USA, 2007. 